\title{
INDICACIONES, HALLAZGOS BRONCOSCÓPICOS Y MORTALIDAD EN PACIENTES PEDIÁTRICOS CON TRAQUEOSTOMÍA EN UN HOSPITAL DE MÉXICO \author{
WITH TRACHEOSTOMY IN A MEXICAN HOSPITAL
} \\ INDICATIONS, BRONCHOSCOPIC FINDINGS AND MORTALITY IN PEDIATRIC PATIENTS
}

\author{
Augusto I Siegert $0^{1}$, Carlos Jaramillo G ${ }^{1}$, Alma D Gonzáles $C^{2}$, Ricardo Alba $P^{2}$, Tania Azcárate $Y^{3}$ \\ 1 Neumólogo pediatra, Residente de broncoscopia pediátrica Hospital del Niño Morelense, Morelos, México \\ 2 Cirujano Pediatra, Hospital del Niño Morelense, Morelos, México \\ 3 Magister en Ciencias Biológicas, Hospital del Niño Morelense, Morelos, México
}

\begin{abstract}
Introduction. Tracheostomy is an old surgical procedure, which is currently performed in pediatric patients. Bronchoscopy is an effective procedure to follow these patients as it reveals data that can not be obtained with other methods. The aim of this study was to determine indications, bronchoscopic findings and mortality in patients with tracheostomy at the Hospital del Niño Morelense (HNM). In addition, we sought to detail the role of bronchoscopy in patients follow-up. Methods. Observational, descriptive and transversal study. We reviewed the records of the patients with tracheostomy during 5 years looking for bronchoscopic findings. Results: The main indication for tracheostomy was prolonged ventilation (74.4\%). The main bronchoscopic diagnoses were subglottic stenosis (20\%), granulomas in the trachea $(70 \%)$, and in bronchi abnormal secretions $(70 \%)$. Mortality was $32.5 \%$. Conclusions: The main indication for tracheostomy is prolonged ventilation. Mortality in children with tracheostomy depends mainly on the underlying pathology. Bronchoscopy is useful for monitoring the child with a tracheostomy.
\end{abstract}

Key words: tracheostomy, bronchoscopy, children

RESUMEN

Introducción. La traqueostomía es un procedimiento quirúrgico antiguo, que se realiza en pediatría cada vez con mayor frecuencia. La broncoscopia es un procedimiento efectivo para el seguimiento del paciente con traqueostomía ya que nos revela datos que no se pueden obtener con otros métodos. Se tuvo como objetivos determinar indicaciones, hallazgos broncoscópicos y mortalidad en pacientes con traqueostomía, en el Hospital del Niño Morelense (HNM). Métodos. Estudio observacional, descriptivo y transversal. Se revisaron los expedientes de los pacientes a quienes se les realizó traqueostomía durante 5 años buscando hallazgos broncoscópicos. Resultados.

La principal indicación de traqueostomía fue la ventilación prolongada (74.4\%). Los principales diagnósticos broncoscópicos fueron: estenosis subglótica (20\%), granulomas en tráquea (70\%) y en bronquios secreciones anormales (70\%). La mortalidad fue de $32.5 \%$. Conclusiones. La principal indicación para traqueostomía fue la ventilación prolongada. La mortalidad en niños con traqueostomía depende principalmente de la patología subyacente. La broncoscopia es útil para el seguimiento del niño con traqueostomía.

Palabras clave: traqueostomia, broncoscopia, niños

\section{INTRODUCCIÓN}

La traqueostomía es uno de los procedimientos quirúrgicos más antiguos reportados en la literatura médica. Se realizó ampliamente en siglos pasados durante las epidemias de difteria y poliomielitis. Mientras que en adultos es un procedimiento aceptado, en niños es percibido como una intervención agresiva ${ }^{(1)}$. Sin embargo, hoy en día las indicaciones para su realización en la edad pediátrica son más frecuentes, siendo las principales: obstrucción de la vía aérea superior, necesi- dad de ventilación prolongada ${ }^{(2)}$ y facilitar la aspiración de secreciones respiratorias en pacientes con enfermedades crónicas.

Un método útil y seguro para el seguimiento del niño con traqueostomía es la broncoscopia. siendo la revisión de la vía aérea en el paciente con traqueostomía una de las indicaciones para realizar este procedimiento ${ }^{(3-5)}$. Este debe realizarse periódicamente para detectar de manera oportuna complicaciones asociadas a la traqueostomía, además se utiliza para evaluar al paciente previo a la decanulación 0 examinar la posición del tubo ${ }^{(3)}$.

\section{Correspondencia:}

Dr Augusto Ignacio Siegert Olivares

Hospital del Niño Morelense

Avenida de la Salud \#1, Morelos - México

Correo electrónico: augustosiegert@gmail.com 
En la literatura se encuentran trabajos sobre indicaciones , complicaciones y mortalidad de niños con traqueostomía $a^{(6-9)}$, sin embargo son pocos los que describen el rol de la broncoscopias en niños con traqueostomía ${ }^{(6,8,9)}$. Por esto la información sobre hallazgos broncoscópicos en este grupo de pacientes es muy escasa. Nuestra investigación tiene como objetivo describir y registrar las indicaciones, hallazgos broncoscópicos y mortalidad de pacientes con traqueostomía, en el Hospital de Niños Morelense durante el periodo 2010 - 2015.

\section{MATERIAL Y MÉTODO}

Estudio observacional, descriptivo y transversal. Se revisaron los expedientes de pacientes con registro de traqueostomía entre marzo 2010 y diciembre de 2015. Se excluyeron quienes se les realizó la traqueostomía en alguna institución de salud ajena al HNM y/o pacientes con expedientes incompletos. Se registró fecha e indicación de la traqueostomía y en el caso que se les haya practicado broncoscopia se determinó la fecha del procedimiento, tiempo transcurrido entre la traqueostomía y las broncoscopias de seguimiento, hallazgos broncoscópicos reportados y estado del paciente al momento del estudio.

Según la edad se clasificó a los pacientes en: neonatos (0-30 días de vida), lactantes (31 días de vida a 1 año 11 meses y 30 días), preescolares (desde los 2 años 0 días hasta los 5 años con 11 meses y 30 días), escolares (desde los 6 años 0 días hasta los 11 años 11 meses y 30 días) y adolescentes (mayores de 12 años). Todas las traqueostomías se hicieron mediante cirugía abierta. La marca de las cánulas usadas fueron Shiley ${ }^{\circledR}$ y Portex ${ }^{\circledR}$. El calibre de las cánulas usadas varió entre 3 y 8 . Las broncoscopias flexibles fueron realizadas con broncoscopios Olympus modelos: BF3C160 y XP160F. Las broncoscopias rígidas fueron realizadas con broncoscopios Storz número 6 en una paciente y número 5 en otro paciente. Se realizaron bajo anestesia general o sedación profunda. Se clasificó como "indeterminado" el estado del paciente si no se halló en el expediente evidencia clara de su muerte y que no haya acudido al hospital en los últimos doce meses antes de la recolección de los datos.

Análisis Estadístico: Para el análisis de la información se usó el programa Epi Info TM 7.1.5.2. Se obtuvo medidas de tendencia central de la muestra y frecuencias simples para las variables de interés.

\section{RESULTADOS}

Se obtuvo un total de 50 reportes de traqueostomía de los cuales 7 presentaban criterios de exclusión, quedando la muestra final conformada por 43 pacientes. El 58.1\% fue de sexo femenino. La media de edad al momento de la realización de traqueostomía fue de 3.9 años, con una desviación estándar de 4.6. Cincuenta por ciento fueron lactantes $(n=22), 21.4 \%$ escolares $(n=9), 19 \%$ preescolares $(n=8)$ y $9.5 \%$ adolescentes $(n=4)$. Al realizar el estudio ningún paciente se encontraba en ventilación domiciliaria. La media de tiempo entre la traqueostomía y la realización de la primera broncoscopia fue de 4.8 meses. En los pacientes que se realizó más de una broncoscopia la media de tiempo entre la realización de las mismas fue de 7 meses.

La principal indicación de traqueostomía fue la necesidad de intubación 0 ventilación mecánica prolongada en el $74.4 \%$ ( $n=32)$, seguida de obstrucción de la vía aérea superior en el 23.2\% ( $n=10)$ y por la necesidad de higiene pulmonar en un paciente (2.3\%). Los diagnósticos para realizar la traqueostomía en el grupo de intubación y/o ventilación prolongada fueron: neumonía grave $(n=13)$, displasia broncopulmonar severa $(n=4)$, tumor intracraneal $(n=4)$, Síndrome de Guillain Barré $(n=2)$, atrofia medular espinal $(n=2)$, traumatismo craneoencefálico $(n=2)$,enfermedad vascular cerebral ( EVC) isquémica $(n=2)$ neuroinfección ( $n=1)$, mielitis transversa $(n=1)$ y EVC hemorrágico $(n=1)$. Los diagnósticos de indicación de traqueostomía en los pacientes del grupo obstrucción de la vía aérea superior fueron: estenosis subglótica $(n=4)$, neoplasias en cuello con compresión de la vía aérea $(n=2)$, intubación orotraqueal difícil $(n=2)$, vía aérea difícil por malformación craneofacial $(n=1)$ y papilomatosis laríngea $(n=1)$. Al momento de la traqueostomía 40 pacientes (93\%) se encontraban en ventilación mecánica. La única paciente cuya indicación para traqueostomía se catalogó como necesidad de higiene pulmonar tenia diagnóstico de parálisis cerebral infantil y la decisión de realizarle traqueostomía, se basó en episodios de apneas asociadas a mal manejo de acumulación de secreciones por deficiencia en los reflejos protectores de la vía aérea, además de neumonía recurrente.

La duración de la traqueostomía en los pacientes que permanecían portando la cánula desde su realización al momento de recolección de los datos fue desde 1 año 0 meses a 4 años 7 meses (media 2.6 años).

Posterior a la traqueostomía se determinó que en el $46.5 \%$ de los pacientes $(n=20)$ se realizó broncoscopia $(n=59)$, siendo la mayoría $(94.9 \%)$ flexibles. Quince de estos pacientes permanecían vivos al momento del estudio, 1 había fallecido y en 4 de ellos no se logró determinar su estado de salud al momento de la investigación. En la a mayoría de los pacientes $(n=15)$ se observaron secreciones anormales en tráquea y bronquios. Se tomaron 13 cultivos de lavado broncoalveolar en 9 pacientes. Los resultados de los cultivos fueron los siguientes: Pseudomona aeruginosa $n=8$, Pseudomona luteola $n=1$, Escherichia coli $n=1$, Candida albicans $n=1$, sin crecimiento $n=2$ En la Tabla 1 se describen los hallazgos broncoscópicos y número de broncoscopías de cada paciente. El paciente con mayor número de procedimientos ( $n=10$ broncoscopías) presentaba una estenosis subglótica y estos tenían como fin realizar dilatación de la vía aérea. Los cuerpos extraños encontrados se trataron de: un diente en bronquio principal izquierdo en un paciente y una semilla en otra paciente con deterioro neurológico y alteración de la mecánica deglutoria. 
Tabla 1. Hallazgos broncoscópicos y número de procedimientos

\begin{tabular}{|c|c|c|}
\hline PACIENTE & NUMERO DE BRONCOSCOPIAS & DIAGNOSTICO ENDOSCOPICO \\
\hline 1 & 4 & Granuloma traqueal \\
\hline 2 & 4 & Laingomalacia \\
\hline 3 & 4 & Laringomalacia, granuloma traqueal, broncomalacia \\
\hline 4 & 6 & Estenosis subglótica, granuloma y traqueobroncomalacia \\
\hline 5 & 1 & Papiloma laríngeo, traqueal y bronquial \\
\hline 6 & 1 & Traquebronco malacia, granuloma traqueal \\
\hline 7 & 10 & Laringomalacia, estenosis subglótica, broncomalacia y estenosis bronquial \\
\hline 8 & 4 & Estenosis subglótica, traqueomalacia \\
\hline 9 & 4 & Estenosis subglótica, traqueomalacia, granuloma traqueal \\
\hline 10 & 7 & Parálisis cuerdas vocales, estenosis suglótica, traqueomalacia, granuloma traqueal, estenosis bronquial \\
\hline 11 & 1 & Traqueomalacia \\
\hline 12 & 1 & Granuloma traqueal \\
\hline 13 & 2 & Traqueomalacia, granuloma traqueal, cuerpo extraño bronquial \\
\hline 14 & 1 & Granuloma traqueal \\
\hline 15 & 1 & Traqueomalacia \\
\hline 16 & 2 & Laringomalacia, bronquiomalacia, granuloma traqueal, \\
\hline 17 & 1 & Granuloma traqueal \\
\hline 18 & 2 & Parálisis cuerdas vocales, granuloma traqueal, cuerpo extraño bronquial \\
\hline 19 & 1 & Granuloma traqueal \\
\hline 20 & 2 & Traqueobroncomalacia, bronqui traqueal, granuloma traqueal \\
\hline
\end{tabular}

Con respecto al estado actual de los pacientes estudiados se determinó que 18 pacientes (41.8\%) se encontraban vivos al momento de la realización del estudio, 9 de ellos decanulados. En 11 pacientes (25.5\%) se consideró su estado actual como "indeterminado". Catorce pacientes (32.5\%) habían fallecido al momento de la investigación, ninguna muerte fue atribuible directamente a la traqueostomía. Las causas de muerte en estos pacientes fueron: choque séptico $(n=4)$, neumonía ( $n=3)$, falla multiorgánica $(n=2)$, insuficiencia respiratoria crónica refractaria $(n=2)$, hipertensión endocraneana $(n=1)$, choque cardiogénico $(n=1)$ y neuroinfección $(n=1)$.

\section{DISCUSIÓN}

En esta investigación encontramos como hallazgos relevantes que la primera indicación para practicar traqueostomia fué la intubación y/o necesidad de ventilacion prolongada principalmente por neumonía. Predominaron los lactantes y el género femenino. En los hallazgos endoscópicos laríngeos, traqueales y bronquiales destacaron: la estenosis subglótica ,los granulomas y las secreciones anormales respectivamente. La mortalidad fue alta en comparación con otros estudios pero las defunciones se debieron a las enfermedades de base de los pacientes.

Las indicaciones para realizar traqueostomía concuerdan con lo reportado por otros autores, con cifras entre $71-87 \%$ para necesidad de apoyo ventilatorio prolongado y $13-20 \%$ para manejo de obstrucción de vía aérea superior ${ }^{7,99}$. También concuerda con lo reportado por Pérez-Ruiz et al, quienes encontraron como principales indicaciones para traqueostomía en su estudio: ventilación mecánica prolongada (62.6\%), estenosis subglótica adquirida (13.6\%), malformaciones craneofaciales (10\%) y anormalidades congénitas de la vía aérea $(9.6 \%){ }^{(8)}$

La similitud de los resultados con los trabajos revisados, se explica por los avances en cuidados críticos pediátricos, que ha llevado a mayor sobrevida en pacientes con patologías graves, donde el asegurar una vía aérea permeable por largos períodos es vital. Esto evidencia el cambio que con el tiempo ha sufrido la indicación para traqueostomía 
en niños la que anteriormente se debía a procesos infecciosos, prácticamente desaparecidos. $^{(1)}$

Con respecto a los hallazgos broncoscópicos en laringe, los casos de laringomalacia y parálisis de cuerdas vocales no fueron la indicación para traqueostomía. Los pacientes con estenosis subglótica se diagnosticaron previo a la traqueostomía y fueron indicación de la misma; esta cifra es similar a lo reportado por Douglas et al ${ }^{(9)}$ quienes describieron estenosis subglótica como indicación para traqueostomía en $14 \%$ de los pacientes y como complicación de la misma en $20 \%$. La similitud con el estudio escocés ${ }^{(9)}$, se debe a la similitud de las poblaciones estudiadas ya que ambos estudios se realizaron en hospitales pediátricos de tercer nivel y, la edad de lactante en ambos estudios fue la más prevalente. Al obtener como principal indicación en el presente estudio para la realización de traqueostomía la intubación y ventilación prolongada, es esperable contar con pacientes con estenosis subglótica ya que la misma principalmente se produce de manera secundaria al trauma laríngeo que genera el tubo orotraqueal, siendo directamente proporcional el riesgo de estenosis subglótica al tiempo de permanencia del tubo ${ }^{(10)}$. Otros factores de riesgo relacionados al desarrollo de estenosis subglótica como dosis de sedantes, intubación traumática, tamaño y material del tubo endotraquea ${ }^{(10,11)}$ son factores que pueden haber contribuido a su desarrollo.

Destaca el alto índice de traqueomalacia (casi la mitad de los pacientes a quienes se les realizó broncoscopia), lo cual podría explicarse por intubación y ventilación mecánica prolongada, que junto con otros factores como altas presiones ventilatorias, biotrauma por oxígeno y la misma presencia de una cánula orotraqueal o de traqueostomía podrían facilitar su desarrollo(12). Llama la atención como hallazgo, un paciente con bronquio traqueal, diagnóstico importante a tomar en cuenta en la evolución de este paciente, por la probabilidad de complicaciones como atelectasias lobares superiores derechas y /o neumonías. ${ }^{(13,14)}$

La incidencia obtenida de granulomas traqueales es semejante a lo reportado por un estudio escocés, quienes reportan sin cifras exactas, que casi todos los pacientes presentaron granulomas estomales, aunque ameritaron intervención sólo 3 de 111 pacientes $^{(9)}$ y a lo reportado por Mahadevan et al (6) quienes describen que los pacientes estudiados tuvieron universalmente tejido de granulación estomal y solo $12.3 \%$ ameritó alguna intervención. La aparición de granulomas se debe a mala posición del tubo y otros factores como el daño repetido por la sonda de aspiración ${ }^{(11,15)}$. La incidencia alta de granulomas traqueales se explica a que en el HNAM no hay un protocolo de revisión periódica de la vía aérea en pacientes portadores de cánulas de traqueostomía, por lo que en muchas ocasiones no se verifica broncoscópicamente la ubicación de la cánula, su distancia con la carina o su contacto con las paredes traqueales. La vía aérea del niño está en pleno desarrollo creciendo tanto en longitud como en diámetro por lo que la misma cánula colocada por largo tiempo, sin monitorear, chocará en algún momento con las paredes traqueales generando tejido de granulación. El tejido de granulación estomal es muy frecuente como se demuestra en el trabajo realizado por Douglas et $a^{(9)}$ quien reportó granuloma estomales en casi todos los pacientes estudiados.

Con respecto a los hallazgos bronquiales reportados por broncoscopia, en la literatura revisada escasean estos datos en pacientes pediátricos con traqueostomía. Si bien estas alteraciones reportadas, necesariamente no son secundarias a la traqueostomía, son ha- llazgos con los que se llega a hacer diagnósticos que pueden cambiar el pronóstico y evolución de los pacientes así como la conducta a tomar por los tratantes. La estenosis bronquial y los granulomas bronquiales (que no se hallaron en el presente trabajo), surgen como complicación frente a la injuria que causa la aspiración repetida e inadecuada de la vía aérea ${ }^{(16)}$. La broncomalacia se produce por injurias múltiples biotrauma por oxígeno, infecciones recurrentes y ventilación con altas presiones $^{(12)}$. El gran número de pacientes con secreciones anormales y la importante incidencia de traqueomalacia pueden explicarse por el efecto de la cánula de traqueostomía, higiene de la misma y el seguimiento deficiente de los pacientes. En la paciente que se reportaron papilomas bronquiales fue la misma que los presentaba en laringe y tráquea. La diseminación traqueobronquial de papilomatosis laríngea se ha asociado al uso de traqueostomía y además el riesgo aumenta mientras mayor tiempo permanezca la cánula. Se sugiere como mecanismo la injuria de la mucosa traqueal causada por el procedimiento, facilita la invasión y multiplicación de papilomas en tráquea y posteriormente en bronquios ${ }^{(17)}$.

La mortalidad de pacientes con traqueostomía en el estudio realizado fue de $32.5 \%$, pudiendo ser mayor por haber pacientes en la muestra con desenlace desconocido, destacándose que ninguna muerte estuvo directamente relacionada con la traqueostomía. Todas las defunciones fueron secundarias a las enfermedades subyacentes de los pacientes. Esta cifra es superior a lo reportado por Mahadevan

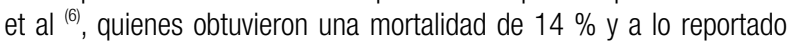
por Douglas et al, quienes reportaron una mortalidad de $21 \%$ en su muestra estudiada ${ }^{(9)}$. Por otra parte es similar a la mortalidad descrita por Atmaca et al $(27.7 \%)^{(7)}$. La mortalidad en niños con traqueostomía es aproximadamente de $40 \%$ y está principalmente relacionada a la patología de base del paciente. La mortalidad directamente asociada al procedimiento de traqueostomía oscila entre $0.5 \%$ y $3 \%{ }^{(3)}$

\section{CONCLUSIONES}

La broncoscopia es un procedimiento seguro para la evaluación periódica de pacientes portadores de traqueostomía y es útil tanto para evaluar complicaciones propias de portar una cánula traqueal como para monitorizar el estado de la patología que condicionó la colocación de la cánula.

Es importante detallar los hallazgos broncoscópicos visualizados, en el seguimiento del paciente con traqueostomía desde la laringe hasta el árbol bronquial; esto debido a que se pueden diagnosticar patologías susceptibles de tratamiento (estenosis laringotraqueales, granulomas, cuerpos extraños) o que ameriten un cambio en el manejo médico (malacias) y, especialmente asegurar la mejor condición del árbol traqueo bronquial previo a un retiro de traqueostomía.

La mortalidad en niños sometidos a traqueostomía depende principalmente de la patología subyacente más que del procedimiento en sí.

\section{Los autores declaran no tener conflicto de interés}




\section{BIBLIOGRAFIA}

1. Barbato A, Bottecchia L, Snijders D. Tracheostomy in children: an ancient procedure still under debate. Eur Respir J. 2012; 40 (6): 1322 $-1323$.

2. Coppola C, Kennedy A, Scorpio R. Pediatric Surgery Diagnosis and Treatment. 2 ed. Suiza: Springer; 2014.

3. Eber E, Oberwaldner B. Tracheostomy care in the hospital. Paediatr Respir Rev. 2006; 7(3): 175 - 184.

4. Pérez-Frías J, Moreno Galdó A, Pérez Ruiz E, Barrio Gómez De Agüero Ml, Escribano Montaner A, Caro Aguilera P. Normativa de Broncoscopia Pediátrica. Arch Bronconeumol 2011; 47 (7):350 - 360.

5. Faro A, Wood R, Schechter M, Leong A, Wittkugel E, Abode K, et al. Official American Thoracic Society Technical Standards: Flexible Airway Endoscopy in Children. Am J Respir Crit Care Med. 2015; 191 (9): $1066-1080$.

6. Mahadevan M, Barber C, Salkeld L, Douglas G, Mills N. Pediatric tracheotomy: 17 year review. Int J Pediatr Otorhinolaryngol. 2007; 71 (12): $1829-1835$.

7. Atmaca S, Bayraktar C, Asilioglu N, Kalkan C, Özsoy Z. Pediatric tracheotomy: 3-year experience at a tertiary care center with 54 children. Turkish J Pediatr 2011; 53: 537 - 540.

8. Perez-Ruiz E, Caro P, Perez-Frías J, Cols M, Barrio I, Torrent A, et al. Paediatric patients with a tracheostomy: a multicentre epidemiological study. Eur Respir J. 2012; 40 (6): 1502 - 1507.

9. Douglas CM, Pooley-Cowley J, Morrissey S, Kuba H, Clement WA, Wynne D. Paediatric tracheostomy-An 11 year experience at a Scottish paediatric tertiary referral centre. Int J Pediatr Otorhinolaryngol.2015; (79): 1673 - 1676.

10. Manica D, Schweiger C, Cauduro PJ, Kuhl G, Antonacci PR. Association Between Length of Intubation and Subglottic Stenosis in Children. Laryngoscope. 2013; 123(4):1049-54.

11. Monnier P. Pediatric Airway Surgery Management of Laryngotracheal Stenosis in Infants and Childrens. Alemania : Springer; 2011.

12. Perillán J. Traqueobroncomalacia en niños. Neumol Pediatr. 2012; 7 (1): 6-12

13. Manjunatha YC Gupta AK. Tracheal Bronchus (Pig Bronchus). Indian J Pediatr. 2010; 77:1037-1038.

14. Pérez JL, Caussade S. Bronquio Traqueal. Neumol Pediatr. 2012; 7 (2): $58-60$

15. López Candiani C, Ceballos Vela MC, Macías HA, Ramírez Candelas B, Rodríguez Weber MA. Estenosis traqueal o bronquial causada por intubación prolongada del recién nacido. Acta Pediatr Mex. 2007; 28(1):9-14.

16. Fisk GC, Baker WDC. Mucosal changes in the trachea and man bronchi of newborn infants after naso-tracheal intubation. Anaesth Intens Care 1975; 3 (3): 209-217.

17. Orji TF, Okorafor IA, Akpeh J0. Experience With Recurrent Respiratory Papillomatosis in a Developing Country: Impact of Tracheostomy. World J Surg. 2013; 37:339-343. 